\title{
Direct Oral Anticoagulants (DOACs) in Cancer Related Thromboembolism
}

\section{Shahid Gilani*}

Cancer Centre, University Hospital of North Midlands, Staffordshire, United Kingdom

*Corresponding Author: Shahid Gilani, Cancer Centre, University Hospital of North Midlands, Staffordshire, United Kingdom.
Received: August 31, 2020

Published: October 01, 2020

(C) All rights are reserved by Shahid Gilani.

\section{Abstract}

Cancer patients have an increased risk of venous thromboembolism (VTE) as well as bleeding. For many years Low Molecule Heparin (LMHW) remained standard of care for these patients. Recently Direct Oral Anticoagulants (DOACs) have shown similar efficacy and safety in large randomised control trials. Data from four recent trials done in cancer patients have highlighted importance of the DOACs in cancer patients. DOACs have been found to be simpler to administer with better compliance among cancer patients. However, there are several issues surrounding prescribing DOACs in these patients. Some of them are related to drug-to-drug interactions and others to an increased risk of bleeding in mucosal cancers of gastrointestinal (GI) and urothelial tracts. In this review, the author summarises updated clinical information and presents an easy to use practical guide for treatment of VTE in cancer patients in a flow-sheet manner. It is also suggested to establish a multidisciplinary cancer associated thrombosis clinic for these patients for long term follow-up.

Keywords: Venous Thromboembolism (VTE); Low Molecule Heparin (LMHW); Cancer Associated Thrombosis (CAT); Direct Oral Anticoagulants (DOACs); Gastrointestinal (GI); Deep Vein Thrombosis (DVT); Pulmonary Embolism (PE)

\section{Introduction}

Thromboembolism covers arterial thrombosis as well as venous thromboembolism (VTE) including deep vein thrombosis (DVT), pulmonary embolism (PE) ${ }^{1}$. VTE is a significant cause of morbidity and mortality in cancer patients [1]. The incidence is even higher during cancer treatment like chemotherapy [2]. The risk of VTE in cancer patients varies from 0.5\% - 20\% depending upon the volume and form of cancer [3], these patients also carry a higher risk of recurrent VTE and bleeding during treatment [4].

Comprehensive management of cancer associated VTE is complex and challenging because of increased risk of bleeding as well as recurrent thrombosis [5]. Drug-to-drug interaction is another concern while on systemic anticancer therapy (SACT) [5]. Since the reporting of the CLOT study in 2003 by Agnes Lee, Low Molecular Weight Heparin (LMWH) remained standard of care in the treatment of cancer-associated thrombosis [6-9]. It has long been agreed that for LMWH is more effective and safer than warfarin in cancer patients with reduced incidence of bleeding and decreased incidence of drug-to-drug interactions [6]. LMWH also remained suitable for patients with poor oral intake as well for those undergoing surgery [6-9].

In recent times evidence has been piling up in favour of Direct Oral Anticoagulants (DOACs) for treatment of VTE in cancer patients 
[10]. Previous studies have established DOACs as a better option than warfarin to treat acute VTE [10]. More recent randomised controlled trials have shown DOACs comparable to LMWH, exhibiting a similar efficacy and safety profile [5]. Moreover, DOACs have a fixed oral dose and predictable anticoagulation effects [5]. DOACs are administered orally avoiding long term subcutaneous injections which improves patient compliance and satisfaction [11].

DOACs display similar efficacy and safety as LMWH in cancer associated thrombosis in head to head randomised controlled trials $[5,13]$. This evidence is shown adequately in four recent randomised studies namely Hokusai VTE Cancer [24], SELECT-D [9], ADAM VTE [13] and Caravaggio trials [16]. This defines an important role of DOACs in the treatment of thrombosis in cancer patients. However, bleeding complications remain a concern particularly in those patients who have active gastro-intestinal and genitourinary malignancies [15]. This has led to recent ASCO [12], NICE [17] and NCCN [33] guidelines updates. Clinical practice guidelines across the world are being updated to include the role of DOACs in the treatment of cancer associated thrombosis [5].

Ambulatory cancer patients on chemotherapy are also at increased risk of VTE and may benefit from pharmacological prophylaxis based on innovative randomised control trials like CASSINI [29] and AVERT [30]. In these trials thrombosis risk stratification is performed based on the Khorana score [15]. High risk patients undergoing chemotherapy with a Khorana score of two or higher may be offered thrombo-prophylaxis [26].

Author has reviewed and summarized current evidence and compiled recommendations for treatment of VTE in cancer patients $[4,12]$. Easy to use flow diagrams are created based on this evidence [4,14-18]. Ongoing and future clinical trial will provide further evidence in this setting. Clinical practice guidelines are being updated to include the role of DOACs in the treatment of thrombosis in cancer patients [5]. Management of cancer associated thrombosis is complex and challenging therefore a longitudinal multidisciplinary cancer associated thrombosis clinic
(CAT-Clinic) is suggested for the long-term follow up of these patients [15].

\section{Current recommendations:}

Treatment of venous thromboembolism (VTE) in cancer patients:

1. Initial treatment may be started with Low Molecular Weight Heparin (LMWH), Unfractionated Heparin (UFH), fondaparinux or Rivaroxaban. Initial 5 - 10 days of LMWH is preferred over UFH for cancer patients without severe renal impairment (Creatinine Clearance $<30 \mathrm{ml} / \mathrm{min}$ ).

2. Long term treatment is recommended for at least 6 months with LMWH, Apixaban, rivaroxaban or edoxaban. Vitamin $\mathrm{K}$ antagonist (warfarin) may be used if LMWH or Direct Oral Anticoagulants (DOACs) are not available. DOACs are associated with increased risk of major bleeding complications particularly in gastrointestinal or genitourinary malignancies. Therefore, careful consideration is needed in choosing DOACs when mucosal bleeding or drug to drug interactions are likely.

3. Long term anticoagulation beyond 6 months may be offered to selected patients with active metastatic disease or in those receiving chemotherapy with a Khorana score of 2 or higher in prophylaxis.

4. Insertion of inferior vena cava filters may not be offered to all patients with established thrombus or to patients with temporary contraindication to anticoagulation. But they may be offered as adjunct to anti-coagulation in those who have developed an extension of an existing thrombus despite optimal therapy.

5. Patients with primary central nervous system (CNS) malignancy or metastatic brain disease with thrombosis may be offered anticoagulation as for other cancers. Although uncertainties do exist around the selection of patients and the approach of anti-coagulation.

6. Incidental PE or DVT should be treated same way as of symptomatic thrombosis. 
7. Treatment of isolated sub-segmental PE or splanchnic or visceral vein thrombi may be considered for treatment on an individual ground.

Prevention of venous thromboembolism (VTE) in cancer patients:

1. Thromboprophylaxis is recommended for hospitalized patients with active cancer and patients undergoing major surgery, in theabsence of bleedingand major contraindications. Routine pharmacologic thromboprophylaxis should not be offered to patients admitted in short stay units for chemotherapy or those undergoing minor procedures like stem cell/ bone marrow transplantation.

2. Patients undergoing chemotherapy should not be offered routine pharmacologic thromboprophylaxis. They should have VTE risk stratification based on the Khorana score. High risk patients undergoing chemotherapy with a Khorana score of 2 or higher may be offered thromboprophylaxis with apixaban, rivaroxaban, or Low Molecular Weight Heparin (LMWH), providing there is no increased risk of bleeding or drug to drug interactions. Discussion should take place with the patient about the risks and benefits including the duration of prophylaxis.

3. Patients with multiple myeloma receiving thalidomide or Lenalidomide should be offered pharmacologic prophylaxis with either aspirin or LMWH for low risk patients and LMWH for high risk patients.

Important considerations when prescribing DOACs in cancer patients

It is important to consider concurrent chemotherapy and other drugs when choosing DOACs. There may be drug to drug interaction with Systemic Anti-Cancer Therapy (SACT) and DOACs. Patient can also develop severe thrombocytopenia during chemotherapy. Moreover, the ability to take medications orally is reduced in some patients due to nausea, vomiting or diarrhoea. Particularly, rivaroxaban appears to be absorbed primarily in the stomach therefore it should be avoided in patients being fed through percutaneous endoscopic jejunostomy (PEJ) [32]. One should have to consider stopping or changing anticoagulation due to intolerance, renal impairment, thrombocytopenia, and drug interaction. DOACs can be withheld or bridged with LMWH when a patient is advised 'Nil by mouth' in peri-operative settings, based on VTE versus bleeding risk evaluation. The assessment of bleeding and recurrent thrombosis risk should continue throughout the treatment. Bleeding complications can occur during treatment requiring blood products and/or reversal of anticoagulation. Decision to reverse DOAC induced anticoagulation may only be considered in critical emergencies. In the majority of cases this should not be necessary due to the short half life of DOACs. Choice of anticoagulation drug should be communicated with the patient at all times even in the palliative care setting and in end of life care.

\section{Conclusion}

The management of VTE in cancer patients is complex and challenging. The majority of current guidelines recommend LMWH as the first line treatment for the initial and long-term management of VTE in cancer patients. However, emerging data from recent randomised controlled trials favour the use of DOACs in this setting. Therefore DOACs are reasonable alternate for patients without an increased risk of bleeding. Although DOACs are convenient and attractive oral options which may increase acceptability to patients, improve patient satisfaction and compliance. However, still many questions remain unanswered. Cancer patients pose unique challenges for anticoagulation in VTE. Many factors need to be considered before choosing the optimal antithrombotic approach. These challenges revolve around active cancer disease, chemotherapy related thrombocytopenia, brain metastases, venous catheter associated thrombosis, low-weight patients, poor oral intake, liver and renal insufficiency, gastrointestinal and urothelial cancers. The management of thrombosis in cancer patients is truly an example of personalised medicine. Addressing these issues in a longitudinal, multidisciplinary cancer associated clinic (CAT Clinic) may be a practical solution. 
Treatment of cancer associated thrombosis (CAT)

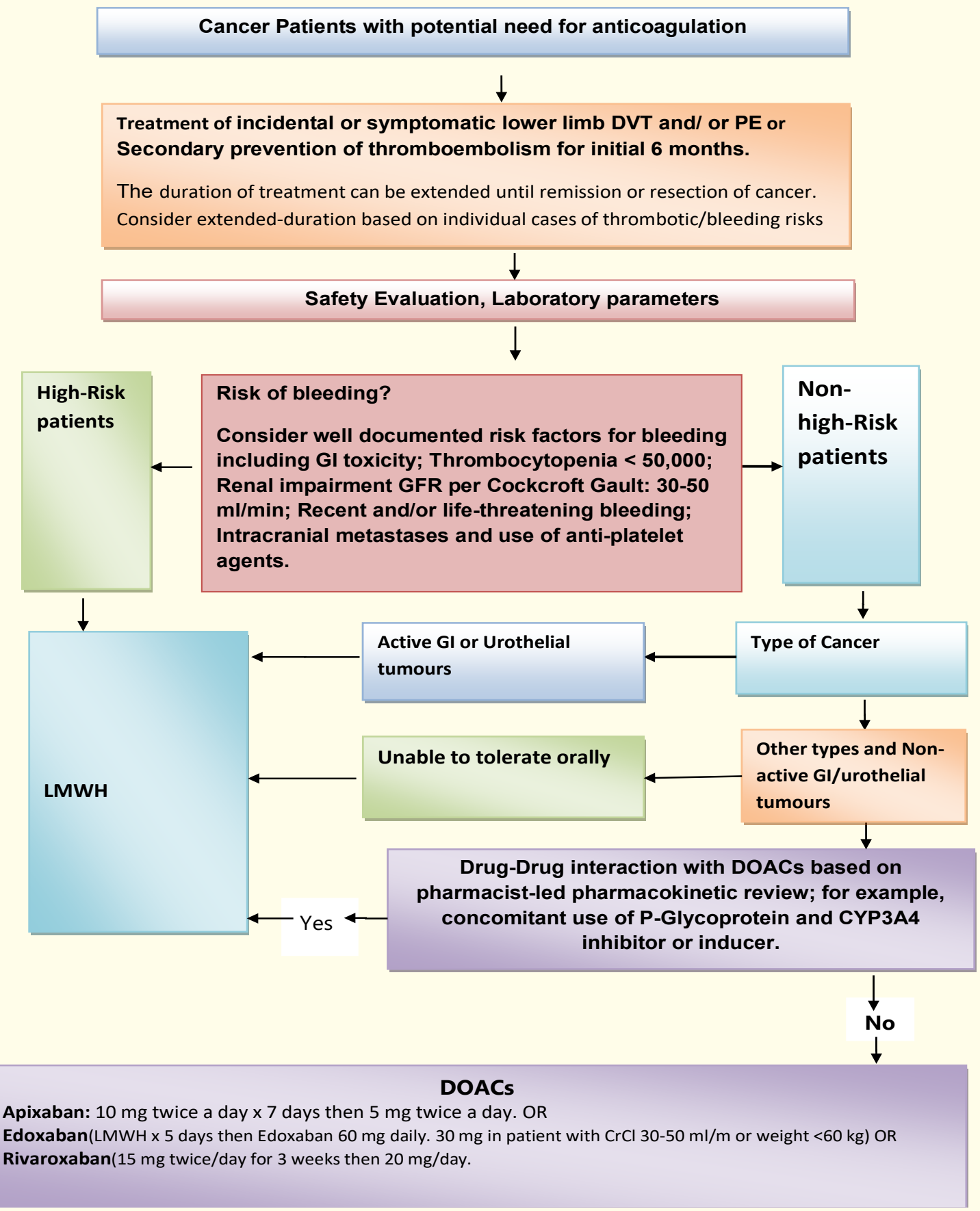

Figure 1: Treatment of Cancer Associated Thrombosis (CAT). 


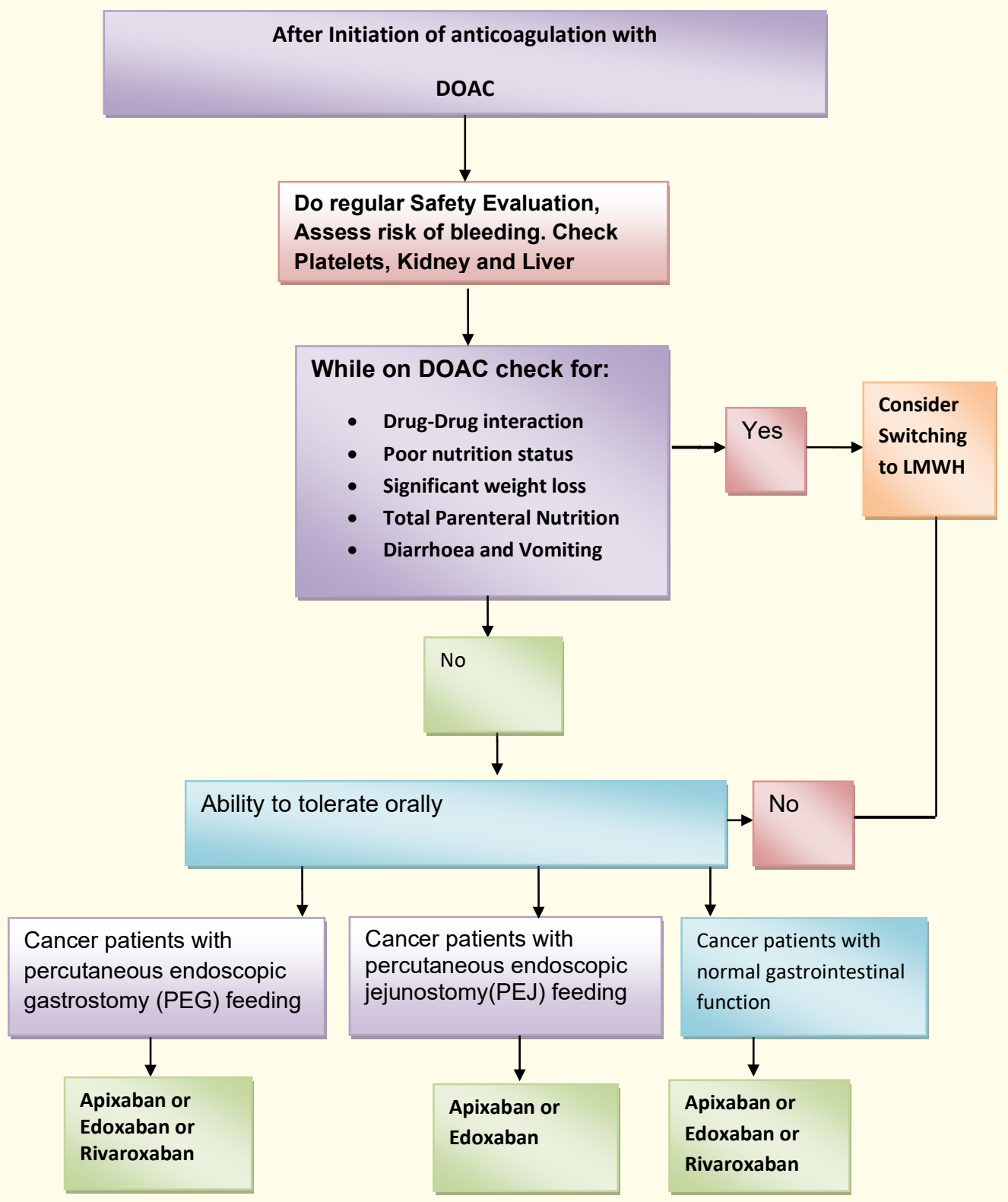

Doses of DOACs: All doses should be taken with food.

Apixaban: $10 \mathrm{mg}$ twice a day $\times 7$ days then $5 \mathrm{mg}$ twice a day. $\mathrm{OR}$

Edoxaban(LMWH x 5 days then Edoxaban $60 \mathrm{mg}$ daily. $30 \mathrm{mg}$ in patient with $\mathrm{CrCl} 30-50 \mathrm{ml} / \mathrm{m}$ or weight $<60 \mathrm{~kg}$ ) OR Rivaroxaban(15 mg twice/day for 3 weeks then $20 \mathrm{mg} /$ day.

Figure 2: Treatment of Cancer Associated Thrombosis (CAT). 
Cancer Patients with liver disease requiring oral anticoagulation (DOAC or Warfarin)

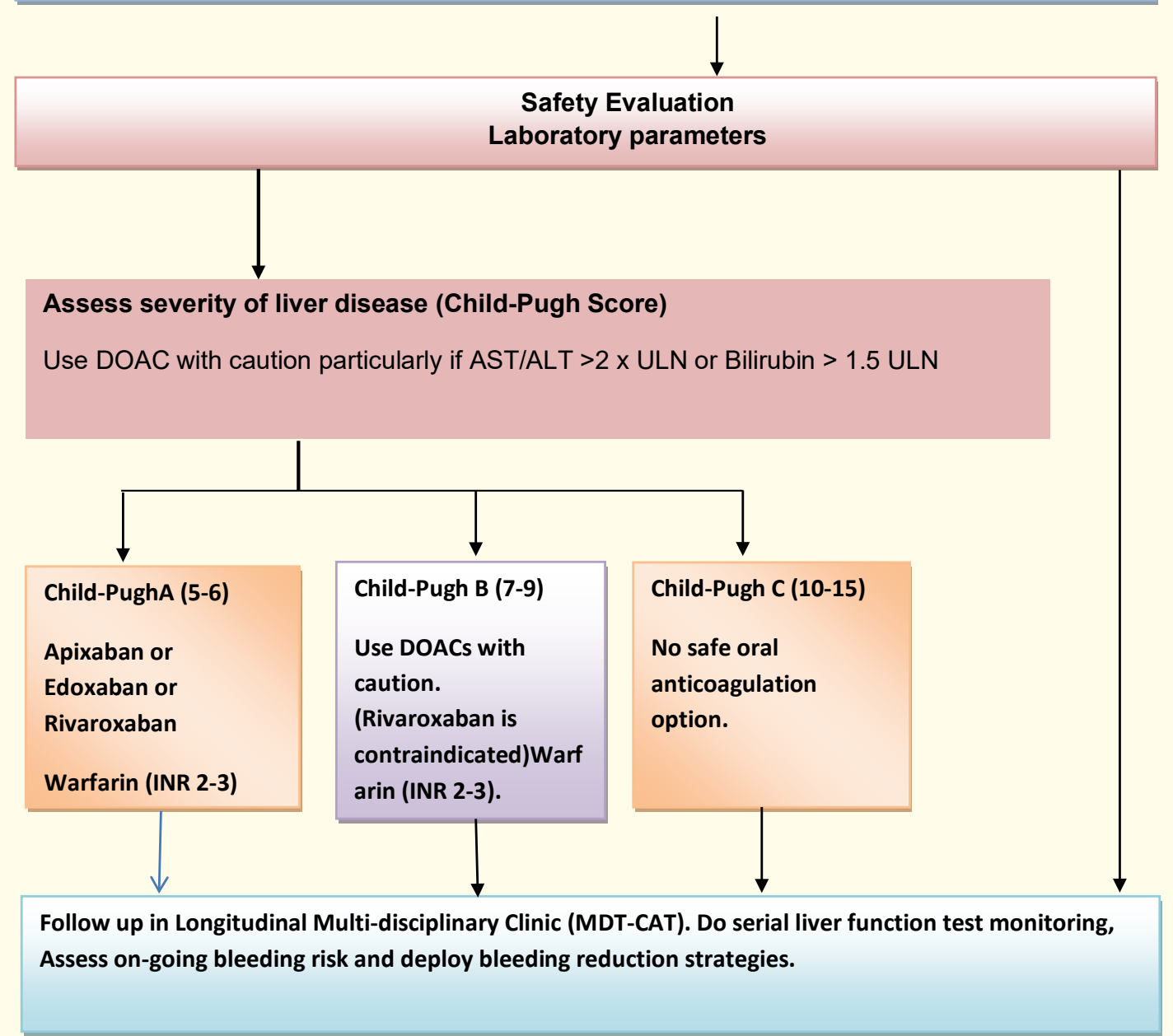

For LMWH/UFH in cancer patients with liver dysfunction, follow standard guidelines

Figure 3: Dose modification of DOACs in Hepatic Insufficiency. 
Dose modification of DOACs in Renal Insufficiency(Table)

\begin{tabular}{|c|c|c|c|}
\hline $\begin{array}{l}\mathrm{Cr} \mathrm{Cl} \\
\text { (Use Cockroft Gault } \\
\text { Creatinine } \\
\text { Clearance instead } \\
\text { of eGFR). }\end{array}$ & Rivaroxaban & $\begin{array}{l}\text { Edoxaban } \\
\text { (Needs } 5 \text { days of } \\
\text { parenteral } \\
\text { anticoagulation) }\end{array}$ & Apixaban \\
\hline $\begin{array}{l}\mathrm{CrCl}>90 \\
\mathrm{ml} / \mathrm{min}\end{array}$ & \multirow[t]{2}{*}{$\begin{array}{l}15 \mathrm{mg} \text { twice a day } \\
\text { for } 21 \text { days, and } \\
\text { then } 20 \mathrm{mg} \text { daily } \\
\text { thereafter }\end{array}$} & $\begin{array}{l}60 \text { mg daily. } \\
\text { But for } \\
\text { patients } \\
\text { weighing <60 } \\
\text { kg } 30 \mathrm{mg} \text { once } \\
\text { daily. }\end{array}$ & $\begin{array}{l}10 \mathrm{mg} \text { twice a } \\
\text { day } x 7 \text { days then } \\
5 \mathrm{mg} \text { twice a day }\end{array}$ \\
\hline $\begin{array}{l}\mathrm{CrCl}>50-90 \\
\mathrm{ml} / \mathrm{min}\end{array}$ & & Same as above & Same as above \\
\hline $\begin{array}{l}\mathrm{Cr} \mathrm{Cl}>30-50 \\
\mathrm{ml} / \mathrm{min}\end{array}$ & 20 mg daily & 30 mg daily & Same as above \\
\hline $\begin{array}{l}\mathrm{CrCl}>15-30 \\
\mathrm{ml} / \mathrm{min}\end{array}$ & $\begin{array}{l}20 \mathrm{mg} \text { daily but } \\
\text { consider } 15 \mathrm{mg} \\
\text { OD if bleeding risk } \\
\text { is high }\end{array}$ & $\begin{array}{l}\text { Not } \\
\text { recommended }\end{array}$ & Use with caution \\
\hline $\begin{array}{l}\mathrm{CrCl}<15 \\
\mathrm{ml} / \mathrm{min}\end{array}$ & $\begin{array}{l}\text { Not } \\
\text { recommended }\end{array}$ & $\begin{array}{l}\text { Not } \\
\text { recommended }\end{array}$ & $\begin{array}{l}\text { Not } \\
\text { recommended }\end{array}$ \\
\hline
\end{tabular}

For LMWH/UFH in cancer patients with renal impairment, follow standard guidelines.

Figure 4: Dose modification of DOACs in Renal Insufficiency. 


\section{Bibliography}

1. Chew HK., et al. "Incidence of venous thromboembolism and its effect on survival among patients with common cancers". Archives of Internal Medicine 166.4 (2006): 458-464.

2. Khorana AA., et al. "Thromboembolism is a leading cause of death in cancer patients receiving outpatient chemotherapy". Journal of Thrombosis and Haemostasis 5.3 (2007): 632-634.

3. Horsted F., et al. "Risk of venous thromboembolism in patients with cancer: a systematic review and meta-analysis". PLOS Medicine 9.7 (2012): e1001275.

4. Nigel S Key., et al. "Venous Thromboembolism Prophylaxis and Treatment in Patients With Cancer: ASCO Clinical Practice Guideline Update”. Journal of Clinical Oncology 38.5 (2020): 496-520.

5. C Ay., et al. "Treatment of Cancer-Associated Venous Thromboembolism in the Age of Direct Oral Anticoagulants". Annals of Oncology 30.6 (2019): 897-907.

6. Farge D., et al. "International clinical practice guidelines including guidance for direct oral anticoagulants in the treatment and prophylaxis of venous thromboembolism in patients with cancer". The Lancet Oncology 17.10 (2016): e452-e466.

7. Mandala M and Labianca R. "European Society for Medical Oncology. Venous thromboembolism (VTE) in cancer patients. ESMO clinical recommendations for prevention and management". Thrombosis Research 125.2 (2010): S117-S119.

8. Kearon C., et al. "Antithrombotic therapy for VTE disease: CHEST guideline and expert panel report". Chest 149.2 (2016): 315-352.

9. Young Annie M., et al. "Comparison of an oral factor Xa inhibitor with low molecular weight heparin in patients with cancer with venous thromboembolism: results of a randomized trial (SELECT-D)". Journal of Clinical Oncology 36.20 (2018): 20172023.
10. Van der Hulle T., et al. "Effectiveness and safety of novel oral anticoagulants as compared with vitamin $\mathrm{K}$ antagonists in the treatment of acute symptomatic venous thromboembolism: a systematic review and meta-analysis". Journal of Thrombosis and Haemostasis 12.3 (2014): 320-328.

11. Wittkowsky AK. "Barriers to the long-term use of low-molecular weight heparins for treatment of cancer-associated thrombosis". Journal of Thrombosis and Haemostasis 4.9 (2006): 2090-2091.

12. ASCO Clinical guidelines for Prevention and Treatment of VTE in Cancer Patients. Journal of Clinical Oncology.

13. McBane R., et al. "Apixaban and Dalteparin in Active Malignancy Associated Venous Thromboembolism: The ADAM VTE Trial". Journal of Thrombosis and Haemostasis (2019): 411-421.

14. Corinine Frere., et al. "Recent Advances in the Management of Cancer-Associated Thrombosis: New Hopes but New Challenges". Cancers 11.1 (2019): 71.

15. Ramya C Mosarla. "Anticoagulation Strategies in Patients with Cancer". Journal of the American College of Cardiology 73 (2019): 1336-1349.

16. Giancarlo Agnelli., et al. "Apixaban for the Treatment of Venous Thromboembolism Associated with Cancer". The New England Journal of Medicine 382 (2020): 1599-1607.

17. NICE. Venous thromboembolic diseases: diagnosis, management and thrombophilia testing. NICE guideline [NG158].

18. Qamar A., et al. "Oral Anticoagulation in Patients with Liver Disease". Journal of the American College of Cardiology 71.19 (2018): 2162-2175.

19. Jens Lutz., et al. "Direct oral anticoagulants in patients with chronic kidney disease: patient selection and special considerations". International Journal of Nephrology and Renovascular Disease 10 (2017): 135-143.

20. David Erskine. DOAC dosing in renal impairment (2020). 
21. Streiff MB., et al. "Venous thromboembolic disease". Journal of the National Comprehensive Cancer Network 11 (2013): 14021429.

22. Lyman GH., et al. "Venous thromboembolism prophylaxis and treatment in patients with cancer: American Society of Clinical Oncology clinical practice guideline update". Journal of Clinical Oncology 31 (2013): 2189-2204.

23. Khorana AA., et al. "Evaluation of US prescription patterns: are treatment guidelines for cancer-associated venous thromboembolism being followed?" Thrombosis Research 145 (2016): 51-53.

24. Raskob GE., et al. "Edoxaban for the treatment of cancer-associated venous thromboembolism". The New England Journal of Medicine (2017).

25. VanEs N., et al. "Edoxaban for treatment of venous thromboembolism in patients with cancer. Rationale and design of the Hokusai VTE-cancer study". Thrombosis and Haemostasis 114 (2015): 1268-1276.

26. Ay C., et al. "Antithrombotic therapy for prophylaxis and treatment of venous thromboembolism in patients with cancer: review of the literature on current practice and emerging options". ESMO Open 2 (2017): e000188.

27. Komatsubara KM and Diuguid DL. "Clotting and bleeding in oncology patients: clinical scenarios and challenges". Oncology 30 (2016): 936-940.

28. Gualandro DM., et al. "II Guidelines for perioperative evaluation of the Brazilian Society of Cardiology". Arquivos Brasileiros de Cardiologia 96.3-1 (2011): 1-68.

29. Khorana Alok A., et al. "Rivaroxaban for Thromboprophylaxis in High-Risk Ambulatory Patients with Cancer". New England Journal of Medicine 380 (2019): 720-728.

30. Carrier Marc., et al. "Apixaban to Prevent Venous Thromboembolism in Patients with Cancer". New England Journal of Medicine 380.8 (2019): 711-719.
31. Adam Cuker., et al. "Reversal of direct oral anticoagulants: Guidance from the Anticoagulation Forum". American Journal of Hematology 94.6 (2019): 697-709.

32. Peterson J., et al. "Administration of Direct Oral Anticoagulants Through Enteral Feeding Tubes". Journal of Pharmacy Technology 32.5 (2016): 196-200.

33. Michael B Streiff., et al. "NCCN Guidelines Insights: CancerAssociated Venous Thromboembolic Disease, Version 2.2018". Journal of the National Comprehensive Cancer Network 16.11 (2018): 1289-1303. 